\title{
Scalar field quasinormal frequencies of Reissner-Nordström black hole surrounded by quintessence by using he continued fraction method
}

\author{
Chen $\mathrm{Wu}^{1 *}$ \\ 1. Shanghai Institute of Applied Physics, \\ Chinese Academy of Sciences, Shanghai 201800, China
}

\begin{abstract}
We evaluate the quasinormal modes of massless scalar field around Reissner-Nordström black hole surrounded by a static and spherically symmetric quintessence by using the continued fraction method. The appropriate Frobenius series for three special cases of the quintessence parameter $\epsilon=-1 / 3,-2 / 3$ and -1 are derived successfully. We show that the variation of quasinormal frequencies with charge of the black hole and the quintessential parameters. The numerical results show that quintessence field decreases oscillation frequencies of all angular momentum $l$ modes and increases the damping time of $l>0$ modes.
\end{abstract}

PACS numbers: $04.30 . \mathrm{Nk}, 04.70 . \mathrm{Bw}, 42.50 . \mathrm{Nn}$

\footnotetext{
* Electronic address: wuchenoffd@gmail.com
} 


\section{INTRODUCTION}

Field perturbations around black holes have been interest objects of discussions for decades. It has been well understood that the decay of fields in black hole background is dominated by some resonant quasinormal modes (QNMs) [1]. The QNMs are defined as the complex solutions to the perturbation wave equations under certain boundary conditions. The study of the QNMs has gained considerable attention coming from AdS/CFT correspondence in string theory. The lowest quasinormal frequencies of black holes have a direct interpretation as dispersion relations of hydrodynamic excitations in the ultra-relativistic heavy ion collisions [2]. Moreover, astrophysical interests in QNMs originated from their relevance in gravitational wave analysis. On September 14th, 2015, two advanced detectors of the Laser Interferometer Gravitational-wave Observatory (LIGO) made the first direct measurement of gravitational waves [3]. The Advanced LIGO detectors observed a transient gravitational-wave signal determined to be the coalescence of two black holes, launching the era of gravitational-wave astronomy. All of these aspects motivated the extensive numerical and analytical study of QNMs for different spacetime and different fields around black holes [4]. We refer the reader to the reviews [5] where a lot of references to the recent research of QNMs can be found.

On the other hand, growing observational evidences indicating our universe are undergoing a stage of accelerated expansion, such as supernova of type Ia [6], the anisotropy of the cosmic microwave background radiation [7], and the large scale structure [8], indicate the presence of some mysterious form of repulsive energy called dark energy. In order to disclose the nature of dark energy, several models have been proposed. The simplest option for this dark energy is the vaccum energy (the Einstein's cosmological constant) having a constant equation of state with state parameter, $\epsilon=-1$, but it needs extreme fine tuning to explain the observations. The second is the dynamical model of dark energy which is often realized by scalar field mechanism (quintessence [9], phantom field [10], and the interaction model between dark energy and dark matter [11]). Different models of dynamical dark energy are determined by the equation of state, namely the ratio of the pressure to the energy density. The quintessence can have an equation of state with $-1 \leq \epsilon \leq-1 / 3$. The cosmological constant with $\epsilon=-1$ can be considered as a special type of the quintessence. If the evolution of the quintessence is slow enough and the kinetic energy density is less than the potential 
energy density, quintessence field can give rise to the negative pressure responsible for the accelerated expansion.

After Kiselev [12] derived the exact solution of Einstein's field equations for quintessential matter surrounding a black hole, many authors considered the evolution of various fields around black holes surrounded by quintessence. Chen and his coworkers calculated the massless scalar field QNMs of the Schwarzschild black hole in the presence of quintessence and observed that all angular momentum $l$ modes damps more rapidly due to the presence of quintessence [13]. While QNMs for other perturbation fields in quintessential Schwarzschild [14] and Reissner-Nordström [15] were calculated. All these studies are based on the calculation of the QNMs by using the third order WKB approximation method.

In this paper, we consider the QNMs of massless scalar field perturbations around Reissner-Nordström black hole surrounded by quintessence by using the continued fraction method. Since it is difficult to analyze the Frobenius series for arbitrary values of the parameter $\epsilon$, we take three special cases of the quintessence parameter, $\epsilon=-1 / 3,-2 / 3$ and -1 , so that the calculations become viable. The continued fraction method proposed by Leaver [16] is considered as the most accurate method to calculate the frequencies of perturbations, since this method has no intermediate approximation compared to other numerical methods. The key ingredient of this method is to cast the perturbation equation into a three-term recurrence relation, and from it we can get a continued fraction equation to work out the QNMs. Though the continued fraction method has been applied to more complex Kerr-Neumann de Sitter background [17], the Frobenius series and the recurrence coefficients are not given explicitly. So it is interesting to implement the continued fraction method to investigate the scalar QNMs of RN-dS spacetime and our study fills this gap. We derive the appropriate Frobenius series for three special cases of the quintessence parameter, $\epsilon=-1 / 3,-2 / 3$ and -1 (this case is the RN-dS spacetime), and then obtain the recurrent relation successfully. We find that the Leaver's method can also be applied to the scalar perturbations of spherically symmetric black hole in de Sitter background.

The rest of the paper is organized as follows. In Sect. 2, we use the continued fraction method to study the scalar perturbations of Reissner-Nordström black hole surrounded by quintessence and the results are presented. The conclusions are given in Sect. 3. 


\section{THE BASIC EQUATIONS AND NUMERICAL RESULTS}

The metric for the spacetime of Reissner-Nordström black hole surrounded by the spherically quintessential matte is given by [12]

$$
d s^{2}=-f(r) d t^{2}+f(r)^{-1} d r^{2}+r^{2} d \theta^{2}+r^{2} \sin ^{2} \theta d \phi^{2}
$$

where

$$
f(r)=1-\frac{2 M}{r}+\frac{Q^{2}}{r^{2}}-\frac{c}{r^{3 \epsilon+1}}
$$

$M$ is the black hole mass, $Q$ is the charge of the black hole, $\epsilon$ represents the state parameter of quintessential and $c$ is the normalization factor which relates to the density of quintessence as, $\rho_{q}=\frac{-c}{2} \frac{3 \epsilon}{r^{3(1-\epsilon)}}$. The Klein-Gordon equation describing the evolution of massless scalar perturbation field outside a charged black hole is given by

$$
\frac{1}{\sqrt{-g}} \partial_{\mu}\left(g^{\mu \nu} \sqrt{-g} \partial_{\nu} \Phi\right)=0
$$

Representing the scalar field in spherical harmonics, one can reduce the wave equation (3) in the background to the Schrödinger wave equation after separation of the angular variables

$$
\left(\frac{d^{2}}{d r^{* 2}}+\omega^{2}-V\left(r^{*}\right)\right) \Psi(r)=0,
$$

with the effective potential $V(r)$ :

$$
V(r)=f(r)\left(\frac{l(l+1)}{r^{2}}+\frac{2 M}{r^{3}}-\frac{2 Q^{2}}{r^{4}}+\frac{c(3 \epsilon+1)}{r^{3 \epsilon+3}}\right),
$$

and

$$
d r^{*}=\frac{d r}{f(r)}
$$

It is difficult to analyze the above wave equation for arbitrary values of the parameter $\epsilon$ by means of the continued fraction method. For our study we take three special cases of the quintessence parameter, $\epsilon=-1 / 3,-2 / 3$ and -1 , so that the calculations become viable. When $c=0$, the effective potential reduces to the one for the Reissner-Nordström spacetime. 


\section{A. the $\epsilon=-1 / 3$ case}

In this case, the black hole horizons are located at $r_{1+}$ and $r_{1-}$, corresponding to zeroes of the function $f(r)$ and we can write the tortoise coordinate as

$$
r_{*}=r+r_{1+} \ln \left(r-r_{1+}\right)+r_{1-} \ln \left(r-r_{1-}\right) .
$$

Within the continued fraction method we can calculate the singular factor of the solution of the wave equation that satisfies in-going wave boundary condition at the event horizon and out-going wave boundary condition at infinity, and expand the remaining part into the Frobenius series that are convergent in the $-\infty<r^{*}<+\infty$ region. The solution of the wave equation Eq. (4) is expanded as follows:

$$
\psi(r)=e^{i \omega r}\left(r-r_{1-}\right)^{i \omega\left(r_{1+}+r_{1-}\right)-1} \sum_{n} a_{n}\left(\frac{r-r_{1+}}{r-r_{1-}}\right)^{n+\delta},
$$

where $\delta=\frac{-i \omega r_{1+}^{2}}{r_{1+}-r_{1-}}$. The coefficients $a_{n}$ satisfy the three- term recurrence relations,

$$
\begin{aligned}
\alpha_{0} a_{1}+\beta_{0} a_{0} & =0 \\
\alpha_{n} a_{n+1}+\beta_{n} a_{n}+\gamma_{n} a_{n-1} & =0, \quad n>0,
\end{aligned}
$$

where

$$
\begin{gathered}
\alpha_{n}=-(n+1)\left[r_{1-}(n+1)+r_{1+}\left(-n+2 i r_{1+} \omega-1\right)\right], \\
\beta_{n}=-r_{1+}\left[l(l+1)+2 n^{2}-4 i r_{1+} \omega(2 n+1)+2 n-8 r_{1+}^{2} \omega^{2}+1\right] \\
+r_{1-}[l(l+1)+2 n(n+1)+1]-2 i(2 n+1) r_{1-} r_{1+} \omega, \\
\gamma_{n}=-\left[n-2 i \omega\left(r_{1+}+r_{1-}\right)\right]\left[n\left(r_{1-}-r_{1+}\right)+2 i r_{1+}^{2} \omega\right] .
\end{gathered}
$$

Since $r_{1+}$ is a regular singular point, convergence of the Frobenius series is automatically satisfied for $r_{1+} \leq r<\infty$. Convergence at $r=\infty$ implies convergence of the sum $\sum_{n} a_{n}$ and the coefficients $a_{n}$ satisfy the following infinite continued fraction equation:

$$
\beta_{0}-\frac{\alpha_{0} \gamma_{1}}{\beta_{1}-} \frac{\alpha_{1} \gamma_{2}}{\beta_{2}-} \cdots=0
$$

Any fundamental QNM can be calculated by solving this nonlinear equation if the infinite continued fraction is truncated at some sufficiently large index. 


\section{B. the $\epsilon=-2 / 3$ case}

The black hole spacetime possesses a cosmological horizon at $r=r_{2 c}$ except for the horizons located at $r_{2+}$ and $r_{2-}$, with $r_{2 c}>r_{2+}>r_{2-}$. Using these horizons $f(r)$ can be written as

$$
f(r)=\frac{c}{r^{2}}\left(r-r_{2+}\right)\left(r-r_{2-}\right)\left(r_{2 c}-r\right)
$$

Introducing the surface gravity $\kappa_{i}=\frac{1}{2}\left|\frac{d f}{d r}\right|_{r=r_{i}}$ associated with the horizon $r=r_{i}$, we can write the analytic form of the tortoise coordinate by calculating Eq. (6):

$$
r_{*}=\frac{1}{2 \kappa_{2+}} \ln \left(\frac{r}{r_{2+}}-1\right)-\frac{1}{2 \kappa_{2-}} \ln \left(\frac{r}{r_{2-}}-1\right)+\frac{1}{2 \kappa_{2 c}} \ln \left(1-\frac{r}{r_{2 c}}\right),
$$

The appropriate Frobenius series which satisfies the boundary conditions can be expanded as

$$
\begin{aligned}
\psi(r)= & \left(\frac{1}{r}-\frac{1}{r_{2+}}\right)^{\frac{i \omega}{2 k_{2+}}}\left(\frac{1}{r}-\frac{1}{r_{2-}}\right)^{-\frac{i \omega}{2 k_{2-}}+\frac{i \omega}{2 k_{2+}}}\left(\frac{1}{r}-\frac{1}{r_{2 c}}\right)^{\frac{i \omega}{2 k_{2 c}}} \\
& \sum_{n} a_{n}\left(\frac{1 / r-1 / r_{2+}}{1 / r-1 / r_{2-}} \frac{1 / r_{2 c}-1 / r_{2-}}{1 / r_{2 c}-1 / r_{2+}}\right)^{n},
\end{aligned}
$$

substituting this expansion into the wave equation, we obtain a six-term recurrence relation for the coefficients $a_{n}$. The Gaussian eliminations allows us to determine the coefficients in the three-term recurrence relation and then scalar QNMs can be solved numerically using standard procedure. For simplicity, we do not list the six-term recurrence relation and the recurrence coefficients in detail.

\section{C. the $\epsilon=-1$ case}

This case corresponds to the Reissner-Nordström-de Sitter (RNdS) spacetime. The zeroes of $f(r)$ are corresponding to the event horizon at $r r_{3+}$, the cauchy horizon at $r_{3-}$, the cosmological horizon at $r_{3 c}$ and a negative zero at $r_{o}=-r_{3-}-r_{3+}-r_{3 c}$, respectively. Then in terms of these horizons and the corresponding surface gravity the tortoise coordinate is defined as

$$
r_{*}=\frac{1}{2 \kappa_{3+}} \ln \left(\frac{r}{r_{3+}}-1\right)-\frac{1}{2 \kappa_{3-}} \ln \left(\frac{r}{r_{3-}}-1\right)-\frac{1}{2 \kappa_{3 c}} \ln \left(1-\frac{r}{r_{3 c}}\right)+\frac{1}{2 \kappa_{o}} \ln \left(1-\frac{r}{r_{o}}\right) .
$$


The appropriate Frobenius series is found to be

$$
\begin{aligned}
\psi(r)= & \left(\frac{1}{r}-\frac{1}{r_{3+}}\right)^{\frac{i \omega}{2 k_{3+}}}\left(\frac{1}{r}-\frac{1}{r_{3-}}\right)^{-\frac{i \omega}{2 k_{3-}}+\frac{i \omega}{2 k_{3+}}}\left(\frac{1}{r}-\frac{1}{r_{3 c}}\right)^{\frac{i \omega}{2 k_{3 c}}}\left(\frac{1}{r}-\frac{1}{r_{o}}\right)^{-\frac{i \omega}{2 k_{o}}} \\
& \sum_{n} a_{n}\left(\frac{1 / r-1 / r_{3+}}{1 / r-1 / r_{3-}} \frac{1 / r_{3 c}-1 / r_{3-}}{1 / r_{3 c}-1 / r_{3+}}\right)^{n},
\end{aligned}
$$

substituting this series into the wave equation we obtain the following seven-term recursion relation for the coefficients $a_{n}$ :

$$
\begin{aligned}
\beta_{0} a_{0}+\alpha_{0} a_{1}=0 \\
\gamma_{1} a_{0}+\beta_{1} a_{1}+\alpha_{1} a_{2}=0 \\
\delta_{2} a_{0}+\gamma_{2} a_{1}+\beta_{2} a_{2}+\alpha_{2} a_{3}=0 \\
\eta_{3} a_{0}+\delta_{3} a_{1}+\gamma_{3} a_{2}+\beta_{3} a_{3}+\alpha_{3} a_{4}=0 \\
\theta_{4} a_{0}+\eta_{4} a_{1}+\delta_{4} a_{2}+\gamma_{4} a_{3}+\beta_{4} a_{4}+\alpha_{4} a_{5}=0 \\
\sigma_{5} a_{0}+\theta_{5} a_{1}+\eta_{5} a_{2}+\delta_{5} a_{3}+\gamma_{5} a_{4}+\beta_{5} a_{5}+\alpha_{5} a_{6}=0 \\
\sigma_{n} a_{n-5}+\theta_{n} a_{n-4}+\eta_{n} a_{n-3}+\delta_{n} a_{n-2}+\gamma_{n} a_{n-1}+\beta_{n} a_{n}+\alpha_{n} a_{n+1}=0
\end{aligned}
$$

where the recurrence coefficients are given in the Appendix. By repeating the Gauss elimination four times, a three-term recurrence relation can be derived. Then the frequencies of the scalar perturbations are the solutions to the characteristic continued fraction equation.

\section{D. numerical results}

Now we report the frequencies of the scalar perturbations in the Reissner-Nordström black holes surrounded by quintessence. It is general experience that the fundamental QNMs will decay slowly and are relevant to the description of scalar field around the black hole. So we consider frequencies of fundamental modes for our study. We obtained the fundamental QNMs for different values of the charge of the black hole Q, the angular momentum index $l$, and the quintessence parameter $\epsilon$. The results are summarized in Table 1.

The dependence of real and imaginary parts of fundamental QNMs on the charge of the black hole is plotted in Fig. 1 for fixed $l=2, c=0.005$ and for different values of $\epsilon$. In this figure, we put the normalization factor, $c=0.005$, so that the deviation of frequencies from the pure Reissner-Nordström spacetime can be clearly understood. The figure shows that the QNMs for scalar field in charged black hole is influenced by quintessence. The 
magnitudes of real and imaginary parts of the $l=2$ modes is lower in the presence of quintessential field. This implies that in the presence of quintessence, the $l=2$ modes for scalar field in Reissner-Nordström black hole damp more slowly. It is found that $\operatorname{Re}(\omega)$ increases monotonically with the increase in $Q$ while the magnitude of $\operatorname{Im}(\omega)$ first decreases, falling to a minimum and thereafter increases. If we switch off the effect of quintessence, our results coincides with those obtained in Ref. [18].

Fig. 2 shows the explicit dependence of $\operatorname{Re}(\omega)$ and $\operatorname{Im}(\omega)$ with quintessential parameter $\epsilon$ and angular momentum $l$ fixed $c=0.005$ and $Q=0.5$. For a fixed $l, R e(\omega)$ increases slightly as the value of $\epsilon$ increases from -1 to $-1 / 3$. The magnitude of $\operatorname{Im}(\omega)$ increases as the value of $\epsilon$ increases for $l>0$ modes while for $l=0$ mode the magnitude of $\operatorname{Im}(\omega)$ first decreases, falling to a smaller value and thereafter increases again.

\section{SUMMARY}

In this paper, we have calculated the QNMs of massless scalar field around ReissnerNordström black hole surrounded by spherically symmetric quintessence by using the continued fraction method. In the extreme case of quintessence $\epsilon=-1$, i.e. the ReissnerNordström-de Sitter (RNdS) spacetime, we have derived a seven-term recurrence relation after Substituting appropriate Frobenius series encoding the QNMs' boundary conditions into the radial equation of scalar field, and then get the characteristic continued fraction equation by means of the the Gauss elimination successfully. We found that the magnitude of $\operatorname{Im}(\omega)$ increases as the value of $\epsilon$ increases for $l>0$ modes while for $l=0$ mode the magnitude of $\operatorname{Im}(\omega)$ first decreases, falling to a smaller value and thereafter increases again.

\section{Acknowledgments}

We thank Prof. Ru-Keng Su for very helpful discussions. This work is supported partially by the Major State Basic Research Development Program in China (No. 2014CB845402). 


\section{Appendix}

In this section, the recurrence coefficients in Eqs. (19) are given explicitly as follow. The quintessence parameter $c$ is taken as $\lambda / 3$ for convenience.

$$
\begin{aligned}
& \alpha_{n}=-(1+n) r_{3+}{ }^{2}\left(r_{3+}-r_{3 c}\right)\left(r_{3-}-r_{3 c}\right)\left(2 r_{3+}+r_{3-}+r_{3 c}\right)^{2} \lambda \\
& \left((1+n)\left(r_{3+}-r_{3-}\right)\left(r_{3+}-r_{3 c}\right)\left(2 r_{3+}+r_{3-}+r_{3 c}\right) \lambda+6 i r_{3+}{ }^{2} \omega\right) \\
& \beta_{n}=r_{3+}\left(-3 l r_{3+}\left(r_{3+}-r_{3-}\right)\left(r_{3+}-r_{3 c}\right)^{2}\left(2 r_{3+}+r_{3-}+r_{3 c}\right)^{2} \lambda-3 l^{2} r_{3+}\left(r_{3+}-r_{3-}\right)\left(r_{3+}-r_{3 c}\right)^{2}\right. \\
& \left(2 r_{3+}+r_{3-}+r_{3 c}\right)^{2} \lambda+4\left(2+n+5 n^{2}\right) r_{3+}{ }^{8} \lambda^{2}+\left(-1-2 n+2 n^{2}\right) r_{3-}{ }^{2} r_{3 c}{ }^{3}\left(r_{3-}+r_{3 c}\right)^{3} \lambda^{2}- \\
& r_{3+} r_{3-} r_{3 c}{ }^{2}\left(r_{3-}+r_{3 c}\right)^{2}\left(\left(-3-5 n+7 n^{2}\right) r_{3-}{ }^{2}+\left(1+2 n-8 n^{2}\right) r_{3-} r_{3 c}-2(1+n)^{2} r_{3 c}{ }^{2}\right) \lambda^{2}- \\
& r_{3+}{ }^{2} r_{3 c}\left(r_{3-}+r_{3 c}\right) \lambda\left(3+4 n-8 n^{2}\right) r_{3-}{ }^{4} \lambda+3\left(-2-3 n+11 n^{2}\right) r_{3-}{ }^{3} r_{3 c} \lambda+\left(1+2 n+4 n^{2}\right) \\
& \left.r_{3 c}{ }^{4} \lambda+r_{3-} r_{3 c}{ }^{2}\left(\left(-2-4 n+n^{2}\right) r_{3 c} \lambda+6 i \omega\right)+r_{3-}{ }^{2} r_{3 c}\left(\left(-2-3 n+11 n^{2}\right) r_{3 c} \lambda+12 i \omega\right)\right)- \\
& r_{3+}{ }^{6}\left(\left(10+7 n+3 n^{2}\right) r_{3-}{ }^{2} \lambda^{2}+2 r_{3-} \lambda\left(n r_{3 c} \lambda+37 n^{2} r_{3 c} \lambda-3 i \omega-78 i n \omega\right)+\left(\left(2+n+5 n^{2}\right)\right.\right. \\
& \left.\left.r_{3 c}{ }^{2} \lambda^{2}+2 i(5+18 n) r_{3 c} \lambda \omega+12 \omega^{2}\right)\right)+r_{3+}{ }^{4}\left(\left(4+3 n-19 n^{2}\right) r_{3-}{ }^{4} \lambda^{2}+3 r_{3-}{ }^{3} \lambda\left(\left(1+2 n+22 n^{2}\right)\right.\right. \\
& \left.r_{3 c} \lambda+2 i(-1+2 n) \omega\right)+3 r_{3-}{ }^{2} r_{3 c} \lambda\left(\left(-1-2 n+14 n^{2}\right) r_{3 c} \lambda-2 i(-5+18 n) \omega\right)+ \\
& 3 r_{3 c}{ }^{2}\left(\left(1+n+3 n^{2}\right) r_{3 c}{ }^{2} \lambda^{2}+8 i(1+3 n) r_{3 c} \lambda \omega+24 \omega^{2}\right)+r_{3-} r_{3 c}\left(\left(-7-10 n+42 n^{2}\right) r_{3 c}{ }^{2} \lambda^{2}\right. \\
& \left.\left.-24 i(-1+n) r_{3 c} \lambda \omega+72 \omega^{2}\right)\right)+r_{3+}{ }^{5}\left(-\left(-1+n+35 n^{2}\right) r_{3-}{ }^{3} \lambda^{2}+r_{3-}{ }^{2} \lambda\left(\left(21+22 n-8 n^{2}\right) r_{3 c} \lambda\right.\right. \\
& +12 i(-1+7 n) \omega)+3 r_{3-}\left(\left(5+5 n+3 n^{2}\right) r_{3 c}^{2} \lambda^{2}-2 i(3+34 n) r_{3 c} \lambda \omega-12 \omega^{2}\right)+ \\
& \left.r_{3 c}\left(\left(11+16 n+38 n^{2}\right) r_{3 c}{ }^{2} \lambda^{2}-18 i(1+4 n) r_{3 c} \lambda \omega+72 \omega^{2}\right)\right)-r_{3+}{ }^{3} \lambda\left(\left(-1-n+3 n^{2}\right) r_{3-}{ }^{5} \lambda\right. \\
& +\left(9+10 n-44 n^{2}\right) r_{3-}{ }^{4} r_{3 c} \lambda+r_{3-} r_{3 c}{ }^{3}\left(\left(9+13 n+13 n^{2}\right) r_{3 c} \lambda-72 i n \omega\right)+ \\
& 2 r_{3-}{ }^{3} r_{3 c}\left(2\left(3+4 n+n^{2}\right) r_{3 c} \lambda+3 i(-3+2 n) \omega\right)+3 r_{3 c}{ }^{4}\left(\left(1+2 n+4 n^{2}\right) r_{3 c} \lambda-4 i(\omega+3 n \omega)\right)+ \\
& \left.r_{3-}{ }^{2} r_{3 c}{ }^{2}\left(4\left(2+3 n+4 n^{2}\right) r_{3 c} \lambda-3 i(\omega+4 n \omega)\right)\right)+r_{3+}{ }^{7} \lambda\left(\left(-1+10 n^{2}\right) r_{3-} \lambda\right. \\
& \left.\left.-3\left(\left(1+n+3 n^{2}\right) r_{3 c} \lambda-i(\omega+6 n \omega)\right)\right)\right) \\
& \gamma_{n}=-\frac{1}{r_{3-}-r_{3 c}}\left(r_{3+}-r_{3 c}\right)\left(-6 l r_{3+}\left(r_{3+}{ }^{2}-{r_{3-}}^{2}\right)\left(r_{3+}-r_{3 c}\right)^{2}\left(2 r_{3+}+r_{3-}+r_{3 c}\right)^{2} \lambda-6 l^{2} r_{3+}\right. \\
& \left(r_{3+}{ }^{2}-r_{3-}{ }^{2}\right)\left(r_{3+}-r_{3 c}\right)^{2}\left(2 r_{3+}+r_{3-}+r_{3 c}\right)^{2} \lambda+20 r_{3+}{ }^{8} r_{3-} \lambda^{2}-16 r_{3+}{ }^{7} r_{3-}{ }^{2} \lambda^{2}-19 r_{3+}{ }^{6} r_{3-}{ }^{3} \lambda^{2} \\
& +7 r_{3+}{ }^{5} r_{3-}{ }^{4} \lambda^{2}+7 r_{3+}{ }^{4} r_{3-}{ }^{5} \lambda^{2}+r_{3+}{ }^{3} r_{3-}{ }^{6} \lambda^{2}-12 r_{3+}{ }^{8} r_{3 c} \lambda^{2}-16 r_{3+}{ }^{7} r_{3-} r_{3 c} \lambda^{2}+27 r_{3+}{ }^{6} r_{3-}{ }^{2} r_{3 c} \lambda^{2} \\
& +28 r_{3+}{ }^{5} r_{3-}{ }^{3} r_{3 c} \lambda^{2}-14 r_{3+}{ }^{4} r_{3-}{ }^{4} r_{3 c} \lambda^{2}-12 r_{3+}{ }^{3} r_{3-}{ }^{5} r_{3 c} \lambda^{2}-r_{3+}{ }^{2} r_{3-}{ }^{6} r_{3 c} \lambda^{2}+3 r_{3+}{ }^{6} r_{3-} r_{3 c}{ }^{2} \lambda^{2} \\
& +6 r_{3+}{ }^{5} r_{3-}{ }^{2} r_{3 c}{ }^{2} \lambda^{2}-17 r_{3+}{ }^{4} r_{3-}{ }^{3} r_{3 c}{ }^{2} \lambda^{2}+3 r_{3+}{ }^{3} r_{3-}{ }^{4} r_{3 c}{ }^{2} \lambda^{2}+6 r_{3+}{ }^{2} r_{3-}{ }^{5} r_{3 c}{ }^{2} \lambda^{2}-r_{3+} r_{3-}{ }^{6} r_{3 c}{ }^{2} \lambda^{2}+
\end{aligned}
$$


$21 r_{3+}{ }^{6} r_{3 c}{ }^{3} \lambda^{2}-12 r_{3+}{ }^{5} r_{3-} r_{3 c}{ }^{3} \lambda^{2}-33 r_{3+}{ }^{4} r_{3-}{ }^{2} r_{3 c}{ }^{3} \lambda^{2}+16 r_{3+}{ }^{3} r_{3-}{ }^{3} r_{3 c}{ }^{3} \lambda^{2}+11 r_{3+}{ }^{2} r_{3-}{ }^{4} r_{3 c}{ }^{3} \lambda^{2}$

$-4 r_{3+} r_{3-}{ }^{5} r_{3 c}{ }^{3} \lambda^{2}+r_{3-}{ }^{6} r_{3 c}{ }^{3} \lambda^{2}+3 r_{3+}{ }^{5} r_{3 c}{ }^{4} \lambda^{2}-14 r_{3+}{ }^{4} r_{3-} r_{3 c}{ }^{4} \lambda^{2}+15 r_{3+}{ }^{3} r_{3-}{ }^{2} r_{3 c}{ }^{4} \lambda^{2}$

$+3 r_{3+}{ }^{2} r_{3-}{ }^{3} r_{3 c}{ }^{4} \lambda^{2}-10 r_{3+} r_{3-}{ }^{4} r_{3 c}{ }^{4} \lambda^{2}+3 r_{3-}{ }^{5} r_{3 c}{ }^{4} \lambda^{2}-9 r_{3+}{ }^{4} r_{3 c}{ }^{5} \lambda^{2}+12 r_{3+}{ }^{3} r_{3-} r_{3 c}{ }^{5} \lambda^{2}$

$+6 r_{3+}{ }^{2} r_{3-}{ }^{2} r_{3 c}{ }^{5} \lambda^{2}-12 r_{3+} r_{3-}{ }^{3} r_{3 c}{ }^{5} \lambda^{2}+3 r_{3-}{ }^{4} r_{3 c}{ }^{5} \lambda^{2}-3 r_{3+}{ }^{3} r_{3 c}{ }^{6} \lambda^{2}+7 r_{3+}{ }^{2} r_{3-} r_{3 c}{ }^{6} \lambda^{2}$

$-5 r_{3+} r_{3-}{ }^{2} r_{3 c}{ }^{6} \lambda^{2}+r_{3-}{ }^{3} r_{3 c}{ }^{6} \lambda^{2}+(-1+n)^{2}\left(r_{3+}-r_{3-}\right)\left(r_{3+}-r_{3 c}\right)^{2}\left(2 r_{3+}+r_{3-}+r_{3 c}\right)^{2}$

$\left(4 r_{3+}{ }^{4}+r_{3+}{ }^{3}\left(20 r_{3-}-6 r_{3 c}\right)-r_{3-}{ }^{2} r_{3 c}\left(r_{3-}+r_{3 c}\right)+r_{3+} r_{3-}\left(3 r_{3-}{ }^{2}-9 r_{3-} r_{3 c}-8 r_{3 c}{ }^{2}\right)\right.$

$\left.+2 r_{3+}{ }^{2}\left(9 r_{3-}{ }^{2}-7 r_{3-} r_{3 c}-3 r_{3 c}{ }^{2}\right)\right) \lambda^{2}+24 i r_{3+}{ }^{8} \lambda \omega+36 i r_{3+}{ }^{7} r_{3-} \lambda \omega-12 i r_{3+}{ }^{6} r_{3-}{ }^{2} \lambda \omega$

$-36 i r_{3+}{ }^{5} r_{3-}{ }^{3} \lambda \omega-12 i r_{3+}{ }^{4} r_{3-}{ }^{4} \lambda \omega-84 i r_{3+}{ }^{7} r_{3 c} \lambda \omega-84 i r_{3+}{ }^{6} r_{3-} r_{3 c} \lambda \omega+60 i r_{3+}{ }^{5} r_{3-}{ }^{2} r_{3 c} \lambda \omega$

$+84 i r_{3+}{ }^{4} r_{3-}{ }^{3} r_{3 c} \lambda \omega+24 i r_{3+}{ }^{3} r_{3-}{ }^{4} r_{3 c} \lambda \omega-48 i r_{3+}{ }^{6} r_{3 c}{ }^{2} \lambda \omega+48 i r_{3+}{ }^{5} r_{3-} r_{3 c}{ }^{2} \lambda \omega$

$+36 i r_{3+}{ }^{4} r_{3-}{ }^{2} r_{3 c}{ }^{2} \lambda \omega-24 i r_{3+}{ }^{3} r_{3-}{ }^{3} r_{3 c}{ }^{2} \lambda \omega-12 i r_{3+}{ }^{2} r_{3-}{ }^{4} r_{3 c}{ }^{2} \lambda \omega+72 i r_{3+}{ }^{5} r_{3 c}{ }^{3} \lambda \omega+$

$24 i r_{3+}{ }^{4} r_{3-} r_{3 c}{ }^{3} \lambda \omega-72 i r_{3+}{ }^{3} r_{3-}{ }^{2} r_{3 c}{ }^{3} \lambda \omega-24 i r_{3+}{ }^{2} r_{3-}{ }^{3} r_{3 c}{ }^{3} \lambda \omega+36 i r_{3+}{ }^{4} r_{3 c}{ }^{4} \lambda \omega$

$-24 i r_{3+}{ }^{3} r_{3-} r_{3 c}{ }^{4} \lambda \omega-12 i r_{3+}{ }^{2} r_{3-}{ }^{2} r_{3 c}{ }^{4} \lambda \omega-72 r_{3+}{ }^{7} \omega^{2}-180 r_{3+}{ }^{6} r_{3-} \omega^{2}-108 r_{3+}{ }^{5} r_{3-}{ }^{2} \omega^{2}$

$+180 r_{3+}{ }^{6} r_{3 c} \omega^{2}+360 r_{3+}{ }^{5} r_{3-} r_{3 c} \omega^{2}+180 r_{3+}{ }^{4} r_{3-}{ }^{2} r_{3 c} \omega^{2}+180 r_{3+}{ }^{5} r_{3 c}{ }^{2} \omega^{2}+180 r_{3+}{ }^{4} r_{3-} r_{3 c}{ }^{2} \omega^{2}+$

$2(-1+n)\left(r_{3+}-r_{3 c}\right)\left(2 r_{3+}+r_{3-}+r_{3 c}\right) \lambda\left(2 r_{3+}{ }^{7} \lambda+r_{3-}{ }^{3} r_{3 c}{ }^{2}\left(r_{3-}+r_{3 c}\right)^{2} \lambda+r_{3+} r_{3-}{ }^{2} r_{3 c}\right.$

$\left(-2 r_{3-}{ }^{3}+r_{3-}{ }^{2} r_{3 c}+4 r_{3-} r_{3 c}{ }^{2}+r_{3 c}{ }^{3}\right) \lambda+r_{3+}{ }^{2} r_{3-}\left(r_{3-}{ }^{4} \lambda-5 r_{3-}{ }^{3} r_{3 c} \lambda\right.$

$\left.-5 r_{3 c}{ }^{4} \lambda+4 r_{3-} r_{3 c}{ }^{2}\left(-2 r_{3 c} \lambda+3 i \omega\right)+r_{3-}{ }^{2} r_{3 c}\left(-5 r_{3 c} \lambda+12 i \omega\right)\right)+r_{3+}{ }^{3}\left(2 r_{3-}{ }^{4} \lambda+2 r_{3-}{ }^{3} r_{3 c} \lambda\right.$

$\left.+3 r_{3 c}{ }^{4} \lambda-4 r_{3-} r_{3 c}{ }^{2}\left(r_{3 c} \lambda+6 i \omega\right)-r_{3-}{ }^{2} r_{3 c}\left(r_{3 c} \lambda+12 i \omega\right)\right)+r_{3+}{ }^{4}\left(-2 r_{3-}{ }^{3} \lambda+12 r_{3-}{ }^{2}\left(r_{3 c} \lambda\right.\right.$

$\left.+4 i \omega)+3 r_{3 c}{ }^{2}\left(2 r_{3 c} \lambda-11 i \omega\right)+r_{3-} r_{3 c}\left(8 r_{3 c} \lambda-57 i \omega\right)\right)+r_{3+}{ }^{6}\left(r_{3-} \lambda-7 r_{3 c} \lambda+18 i \omega\right)$

$\left.\left.-r_{3+}{ }^{5}\left(4 r_{3-}{ }^{2} \lambda+r_{3 c}\left(4 r_{3 c} \lambda+33 i \omega\right)-69 i r_{3-} \omega\right)\right)\right)$

$$
\begin{aligned}
\delta_{n}= & \frac{1}{\left(r_{3-}-r_{3 c}\right)^{2}}\left(r_{3+}-r_{3 c}\right)^{2}\left(-3 l\left(r_{3+}{ }^{3}+3 r_{3+}{ }^{2} r_{3-}-3 r_{3+} r_{3-}{ }^{2}-r_{3-}{ }^{3}\right)\left(r_{3+}-r_{3 c}\right)^{2}\right. \\
& \left(2 r_{3+}+r_{3-}+r_{3 c}\right)^{2} \lambda-3 l^{2}\left(r_{3+}{ }^{3}+3 r_{3+}{ }^{2} r_{3-}-3 r_{3+} r_{3-}{ }^{2}-r_{3-}{ }^{3}\right)\left(r_{3+}-r_{3 c}\right)^{2} \\
& \left(2 r_{3+}+r_{3-}+r_{3 c}\right)^{2} \lambda+(-2+n)^{2}\left(r_{3+}-r_{3-}\right)\left(r_{3+}-r_{3 c}\right)^{2}\left(2 r_{3+}+r_{3-}+r_{3 c}\right)^{2} \\
& \left(r_{3+}{ }^{4}+2 r_{3+}{ }^{3}\left(7 r_{3-}-2 r_{3 c}\right)+2 r_{3+} r_{3-}\left(7 r_{3-}{ }^{2}-8 r_{3-} r_{3 c}-6 r_{3 c}{ }^{2}\right)+r_{3-}{ }^{2}\left(r_{3-}{ }^{2}-4 r_{3-} r_{3 c}-4 r_{3 c}{ }^{2}\right)+\right. \\
& \left.r_{3+}{ }^{2}\left(15 r_{3-}{ }^{2}-8 r_{3-} r_{3 c}-2 r_{3 c}{ }^{2}\right)\right) \lambda^{2}+(-2+n)\left(r_{3+}-r_{3 c}\right)\left(2 r_{3+}+r_{3-}+r_{3 c}\right) \lambda \\
& \left(2 r_{3+}{ }^{7} \lambda+r_{3-}{ }^{3} r_{3 c}\left(-r_{3-}{ }^{3}+5 r_{3-}{ }^{2} r_{3 c}+12 r_{3-} r_{3 c}{ }^{2}+6 r_{3 c}{ }^{3}\right) \lambda+r_{3+}{ }^{2} r_{3-}\left(5 r_{3-}{ }^{4} \lambda\right.\right. \\
& \left.-6 r_{3 c}{ }^{4} \lambda-24 r_{3-} r_{3 c}{ }^{2}\left(r_{3 c} \lambda-i \omega\right)-r_{3-}{ }^{3}\left(11 r_{3 c} \lambda+12 i \omega\right)+2 r_{3-}{ }^{2} r_{3 c}\left(-7 r_{3 c} \lambda+24 i \omega\right)\right)- \\
& r_{3+}{ }^{5}\left(5 r_{3-}{ }^{2} \lambda+10 r_{3-}\left(r_{3 c} \lambda-12 i \omega\right)+r_{3 c}\left(7 r_{3 c} \lambda+48 i \omega\right)\right)+r_{3+}{ }^{4}\left(-12 r_{3-}{ }^{3} \lambda+12 r_{3 c}{ }^{2}\left(r_{3 c} \lambda-4 i \omega\right)\right.
\end{aligned}
$$


$\left.+3 r_{3-} r_{3 c}\left(3 r_{3 c} \lambda-56 i \omega\right)+r_{3-}{ }^{2}\left(25 r_{3 c} \lambda+192 i \omega\right)\right)+r_{3+}{ }^{6}\left(7 r_{3-} \lambda-13 r_{3 c} \lambda+12 i \omega\right)$

$r_{3+}{ }^{6}\left(7 r_{3-} \lambda-13 r_{3 c} \lambda+12 i \omega\right)+r_{3+} r_{3-}{ }^{2}\left(r_{3-}{ }^{4} \lambda-10 r_{3-}{ }^{3} r_{3 c} \lambda-6 r_{3 c}{ }^{4} \lambda-3 r_{3-}{ }^{2} r_{3 c}\left(r_{3 c} \lambda-8 i \omega\right)+\right.$ $\left.24 i r_{3-} r_{3 c}{ }^{2} \omega\right)+r_{3+}{ }^{3}\left(r_{3-}{ }^{4} \lambda+3 r_{3 c}{ }^{4} \lambda+2 r_{3-}{ }^{3}\left(5 r_{3 c} \lambda+12 i \omega\right)+r_{3-}{ }^{2} r_{3 c}\left(5 r_{3 c} \lambda-48 i \omega\right)-\right.$

$\left.\left.-60 i r_{3-} r_{3 c}{ }^{2} \omega\right)\right) 3\left(-r_{3-}{ }^{3} r_{3 c}{ }^{3}\left(r_{3-}+r_{3 c}\right)^{3} \lambda^{2}+r_{3+} r_{3-}{ }^{2} r_{3 c}{ }^{2}\left(r_{3-}+r_{3 c}\right)^{2}\left(r_{3-}{ }^{2}+3 r_{3 c}{ }^{2}\right) \lambda^{2}+\right.$

$r_{3+}{ }^{2} r_{3-} r_{3 c}\left(r_{3-}+r_{3 c}\right) \lambda\left(r_{3-}{ }^{4} \lambda-3 r_{3 c}{ }^{4} \lambda+r_{3-}{ }^{3}\left(3 r_{3 c} \lambda-2 i \omega\right)+4 r_{3-}{ }^{2} r_{3 c}\left(r_{3 c} \lambda+3 i \omega\right)+3 r_{3-} r_{3 c}{ }^{2}\right.$

$\left.\left(r_{3 c} \lambda+4 i \omega\right)\right)+4 r_{3+}{ }^{8} \lambda\left(r_{3-} \lambda+r_{3 c} \lambda-i \omega\right)-2 r_{3+}{ }^{7}\left(4 r_{3-}{ }^{2} \lambda^{2}+r_{3-} \lambda\left(4 r_{3 c} \lambda+9 i \omega\right)+\left(-13 i r_{3 c} \lambda-\right.\right.$ $-6 \omega) \omega) r_{3+}{ }^{3} r_{3-}{ }^{6} \lambda^{2}+6 r_{3-} r_{3 c}{ }^{5} \lambda^{2}-r_{3 c}{ }^{6} \lambda^{2}+2 r_{3-}{ }^{5} \lambda\left(r_{3 c} \lambda-i \omega\right)+r_{3-}{ }^{4} r_{3 c} \lambda\left(9 r_{3 c} \lambda+22 i \omega\right)+$ $\left.r_{3-}{ }^{3} r_{3 c}\left(4 r_{3 c}{ }^{2} \lambda^{2}-i r_{3 c} \lambda \omega+12 \omega^{2}\right)+3 r_{3-}{ }^{2} r_{3 c}{ }^{2}\left(5 r_{3 c}{ }^{2} \lambda^{2}-8 i r_{3 c} \lambda \omega+16 \omega^{2}\right)\right)+$ $r_{3+}{ }^{5} 5 r_{3-}{ }^{4} \lambda^{2}+2 r_{3-}{ }^{3} \lambda\left(5 r_{3 c} \lambda+8 i \omega\right)+2 r_{3-} r_{3 c}\left(7 r_{3 c}{ }^{2} \lambda^{2}-2 i r_{3 c} \lambda \omega-96 \omega^{2}\right)+$ $\left.r_{3-}{ }^{2}\left(5 r_{3 c}{ }^{2} \lambda^{2}-i r_{3 c} \lambda \omega+27 \omega^{2}\right)-r_{3 c}{ }^{2}\left(r_{3 c}{ }^{2} \lambda^{2}+24 i r_{3 c} \lambda \omega+48 \omega^{2}\right)\right)+r_{3+}{ }^{6}\left(r_{3-}{ }^{3} \lambda^{2}\right.$ $\left.-r_{3-}{ }^{2} \lambda\left(r_{3 c} \lambda+8 i \omega\right)+r_{3 c}\left(-7 r_{3 c}{ }^{2} \lambda^{2}+14 i r_{3 c} \lambda \omega-48 \omega^{2}\right)+r_{3-}\left(-9 r_{3 c}{ }^{2} \lambda^{2}+46 i r_{3 c} \lambda \omega+84 \omega^{2}\right)\right)-$ $r_{3+}{ }^{4}\left(r_{3-}{ }^{5} \lambda^{2}+4 r_{3-}{ }^{4} \lambda\left(r_{3 c} \lambda-3 i \omega\right)-3 r_{3 c}{ }^{4} \lambda\left(r_{3 c} \lambda-4 i \omega\right)+r_{3-}{ }^{3}\left(7 r_{3 c}{ }^{2} \lambda^{2}+44 i r_{3 c} \lambda \omega-36 \omega^{2}\right)-\right.$ $\left.\left.\left.r_{3-} r_{3 c}^{2}\left(r_{3 c}^{2} \lambda^{2}-3 i r_{3 c} \lambda \omega-18 \omega^{2}\right)+r_{3-}{ }^{2} r_{3 c}\left(-r_{3 c}{ }^{2} \lambda^{2}+24 i r_{3 c} \lambda \omega+192 \omega^{2}\right)\right)\right)\right)$

$$
\begin{aligned}
& \eta_{n}=\frac{1}{\left(r_{3-}-r_{3 c}\right)^{3}}\left(r_{3+}-r_{3 c}\right)^{3}\left(6 l r_{3-}\left(r_{3+}{ }^{2}-r_{3-}{ }^{2}\right)\left(r_{3+}-r_{3 c}\right)^{2}\left(2 r_{3+}+r_{3-}+r_{3 c}\right)^{2} \lambda\right. \\
& +6 l^{2} r_{3-}\left(r_{3+}{ }^{2}-r_{3-}{ }^{2}\right)\left(r_{3+}-r_{3 c}\right)^{2}\left(2 r_{3+}+r_{3-}+r_{3 c}\right)^{2} \lambda-4(-2+n) r_{3+}{ }^{8}\left((-10+3 n) r_{3-}\right. \\
& \left.-(-2+n) r_{3 c}\right) \lambda^{2}-r_{3+} r_{3-}{ }^{2} r_{3 c}\left(r_{3-}+r_{3 c}\right) \lambda\left(-r_{3 c}\left(11 r_{3-}{ }^{3}+9 r_{3-}{ }^{2} r_{3 c}+5 r_{3-} r_{3 c}{ }^{2}+7 r_{3 c}{ }^{3}\right) \lambda\right. \\
& +2(-3+n)^{2}\left(4 r_{3-}{ }^{4}-18 r_{3-}{ }^{3} r_{3 c}-4 r_{3-}{ }^{2} r_{3 c}{ }^{2}+11 r_{3-} r_{3 c}{ }^{3}+r_{3 c}{ }^{4}\right) \lambda+(-3+n) \\
& \left.\left(-2 r_{3-}{ }^{4} \lambda+5 r_{3 c}{ }^{4} \lambda+r_{3-}{ }^{3}\left(8 r_{3 c} \lambda+6 i \omega\right)+2 r_{3-}{ }^{2} r_{3 c}\left(r_{3 c} \lambda-15 i \omega\right)+r_{3-} r_{3 c}{ }^{2}\left(r_{3 c} \lambda-24 i \omega\right)\right)\right)- \\
& 8 r_{3+}{ }^{7} \lambda\left(\left(76-53 n+9 n^{2}\right) r_{3-}{ }^{2} \lambda-3 i(-2+n) r_{3 c} \omega+r_{3-}\left(-2\left(20-16 n+3 n^{2}\right) r_{3 c} \lambda+\right.\right. \\
& +3 i(-8+3 n) \omega)) r_{3-}{ }^{3} r_{3 c}{ }^{2}\left(r_{3-}+r_{3 c}\right)^{2} \lambda\left(-3 r_{3 c}\left(r_{3-}+r_{3 c}\right) \lambda+2(-3+n)^{2}\left(2 r_{3-}{ }^{2}-3 r_{3-} \lambda\right.\right. \\
& \left.\left.r_{3 c}-3 r_{3 c}{ }^{2}\right)+(-3+n)\left(-2{r_{3-}}^{2} \lambda+6 r_{3-} r_{3 c} \lambda+6 r_{3 c}{ }^{2} \lambda+6 i r_{3-} \omega\right)\right)+r_{3+}{ }^{2} r_{3-}\left(2\left(21-13 n+2 n^{2}\right)\right. \\
& r_{3-}{ }^{6} \lambda^{2}+\left(52-40 n+7 n^{2}\right) r_{3 c}{ }^{6} \lambda^{2}+3 r_{3-} r_{3 c}{ }^{4} \lambda\left(\left(71-38 n+5 n^{2}\right) r_{3 c} \lambda-12 i(-4+n) \omega\right)+ \\
& r_{3-}{ }^{5} \lambda\left(\left(-625+390 n-62 n^{2}\right) r_{3 c} \lambda+6 i(-3+n) \omega\right)+2 r_{3-}{ }^{4} r_{3 c} \lambda\left(\left(161-102 n+16 n^{2}\right) r_{3 c} \lambda-\right. \\
& 6 i(-20+7 n) \omega)+r_{3-}{ }^{3} r_{3 c}\left(( 9 2 8 - 5 8 0 n + 9 1 n ^ { 2 } ) r _ { 3 c } { } ^ { 2 } \lambda ^ { 2 } r _ { 3 - } { } ^ { 2 } r _ { 3 c } { } ^ { 2 } \left(\left(268-138 n+17 n^{2}\right)\right.\right. \\
& \left.\left.r_{3 c}{ }^{2} \lambda^{2}+24 i n r_{3 c} \lambda \omega-36 \omega^{2}\right)\right)+r_{3+}{ }^{3}\left(\left(317-200 n+32 n^{2}\right) r_{3-}{ }^{6} \lambda^{2}+(-2+n)^{2} r_{3 c}{ }^{6} \lambda^{2}-\right. \\
& 4 r_{3-}{ }^{5} \lambda\left(5\left(67-43 n+7 n^{2}\right) r_{3 c} \lambda+3 i(5-2 n) \omega\right)+12 r_{3-} r_{3 c}{ }^{4} \lambda\left(\left(-4+2(-3+n)^{2}+n\right) r_{3 c} \lambda\right. \\
& -2 i(-11+4 n) \omega)+3 r_{3-}{ }^{2} r_{3 c}{ }^{2}\left(\left(23-28 n+6 n^{2}\right) r_{3 c}{ }^{2} \lambda^{2}-8 i(-34+11 n) r_{3 c} \lambda \omega-108 \omega^{2}\right)+
\end{aligned}
$$


$4 r_{3-}{ }^{3} r_{3 c}\left(\left(-9-16 n+5 n^{2}\right) r_{3 c}{ }^{2} \lambda^{2}-18 i(-3+n) r_{3 c} \lambda \omega-90 \omega^{2}\right)-r_{3-}{ }^{4}\left(\left(122-64 n+9 n^{2}\right)\right.$

$\left.\left.r_{3 c}{ }^{2} \lambda^{2}+4 i(-1+2 n) r_{3 c} \lambda \omega-12 \omega^{2}\right)\right)-r_{3+}{ }^{6}\left(\left(704-440 n+71 n^{2}\right) r_{3-}{ }^{3} \lambda^{2}+r_{3-}{ }^{2} \lambda\right.$

$\left(\left(-1160+788 n-131 n^{2}\right) r_{3 c} \lambda+36 i(-26+9 n) \omega\right)+r_{3 c}\left(7(-2+n)^{2} r_{3 c}{ }^{2} \lambda^{2}-\right.$

$\left.\left.12 i(-2+n) r_{3 c} \lambda \omega+36 \omega^{2}\right)-r_{3-}\left(\left(12-8 n+n^{2}\right) r_{3 c}{ }^{2} \lambda^{2}+8 i(-29+11 n) r_{3 c} \lambda \omega+36 \omega^{2}\right)\right)+$

$r_{3+}{ }^{5}\left(\left(308-230 n+41 n^{2}\right) r_{3-}{ }^{4} \lambda^{2}+4 r_{3-}{ }^{3} \lambda\left(\left(276-164 n+25 n^{2}\right) r_{3 c} \lambda-3 i(-91+30 n) \omega\right)-\right.$

$r_{3 c}^{2}\left((-2+n)^{2} r_{3 c}{ }^{2} \lambda^{2}+24 i(-2+n) r_{3 c} \lambda \omega+36 \omega^{2}\right)-4 r_{3-} r_{3 c}\left(\left(110-87 n+16 n^{2}\right) r_{3 c}{ }^{2} \lambda^{2}\right.$

$\left.-6 i(-14+5 n) r_{3 c} \lambda \omega+90 \omega^{2}\right)+r_{3-}{ }^{2}\left(-2\left(-28+n+2 n^{2}\right) r_{3 c}{ }^{2} \lambda^{2}+12 i(-135+46 n) r_{3 c} \lambda \omega\right.$

$\left.\left.+324 \omega^{2}\right)\right)+r_{3+}{ }^{4}\left(\left(725-472 n+78 n^{2}\right) r_{3-}{ }^{5} \lambda^{2}+3(-2+n) r_{3 c}{ }^{4} \lambda\left((-2+n) r_{3 c} \lambda-4 i \omega\right)+\right.$

$r_{3-}{ }^{4} \lambda\left(\left(-551+414 n-73 n^{2}\right) r_{3 c} \lambda+12 i(24-7 n) \omega\right)-2 r_{3-} r_{3 c}{ }^{2}\left(\left(28-20 n+3 n^{2}\right) r_{3 c}{ }^{2} \lambda^{2}\right.$

$\left.+36 i(-8+3 n) r_{3 c} \lambda \omega+162 \omega^{2}\right)+r_{3-}{ }^{3}\left(\left(-389+244 n-36 n^{2}\right) r_{3 c}{ }^{2} \lambda^{2}+12 i(-97+30 n) r_{3 c} \lambda \omega\right.$

$\left.\left.\left.+252 \omega^{2}\right)-r_{3-}{ }^{2} r_{3 c}\left(\left(909-562 n+86 n^{2}\right) r_{3 c}{ }^{2} \lambda^{2}-12 i(-23+6 n) r_{3 c} \lambda \omega+648 \omega^{2}\right)\right)\right)$

$\theta_{n}=\frac{1}{\left(r_{3-}-r_{3 c}\right)^{4}} r_{3-}\left(r_{3+}-r_{3 c}\right)^{4}\left(-3 l\left(r_{3+}-r_{3-}\right) r_{3-}\left(r_{3+}-r_{3 c}\right)^{2}\left(2 r_{3+}+r_{3-}+r_{3 c}\right)^{2} \lambda\right.$

$-3 l^{2}\left(r_{3+}-r_{3-}\right) r_{3-}\left(r_{3+}-r_{3 c}\right)^{2}\left(2 r_{3+}+r_{3-}+r_{3 c}\right)^{2} \lambda+4 r_{3+}{ }^{7}\left(\left(43-23 n+3 n^{2}\right) r_{3-}\right.$

$\left.+\left(-23+14 n-2 n^{2}\right) r_{3 c}\right) \lambda^{2}-r_{3-}{ }^{2} r_{3 c}{ }^{2}\left(r_{3-}+r_{3 c}\right)^{2} \lambda\left(\left(86-41 n+5 n^{2}\right) r_{3-}{ }^{2} \lambda\right.$

$\left.+\left(-73+34 n-4 n^{2}\right) r_{3 c}^{2} \lambda+r_{3-}\left(\left(-73+34 n-4 n^{2}\right) r_{3 c} \lambda+12 i(-4+n) \omega\right)\right)+$

$r_{3+} r_{3-} r_{3 c}\left(r_{3-}+r_{3 c}\right) \lambda\left(2\left(86-41 n+5 n^{2}\right) r_{3-}{ }^{4} \lambda-2(-5+n)^{2} r_{3 c}{ }^{4} \lambda+r_{3-} r_{3 c}{ }^{2}\left(\left(119-62 n+8 n^{2}\right)\right.\right.$

$\left.r_{3 c} \lambda-24 i(-4+n) \omega\right)+r_{3-}{ }^{3}\left(\left(-391+189 n-23 n^{2}\right) r_{3 c} \lambda+24 i(-4+n) \omega\right)+r_{3-}{ }^{2} r_{3 c}((-2-3$

$\left.\left.\left.(-4+n)^{2}+n\right) r_{3 c} \lambda-48 i(-4+n) \omega\right)\right)+4 r_{3+}{ }^{6} \lambda\left(\left(-1+10(-4+n)^{2}\right) r_{3-}{ }^{2} \lambda-6 i(-7+2 n) r_{3 c}\right.$

$\left.\omega+r_{3-}\left(-\left(-5+9(-4+n)^{2}+n\right) r_{3 c} \lambda+3 i(-23+6 n) \omega\right)\right)+r_{3+}{ }^{2}\left(\left(-86+41 n-5 n^{2}\right) r_{3-}{ }^{6} \lambda^{2}\right.$

$r_{3 c}{ }^{6}+\left(-23+14 n-2 n^{2}\right) \lambda^{2}+r_{3-}{ }^{5} \lambda\left(\left(735-358 n+44 n^{2}\right) r_{3 c} \lambda-12 i(-4+n) \omega\right)-$

$3 r_{3-} r_{3 c}{ }^{4} \lambda\left(\left(73-34 n+4 n^{2}\right) r_{3 c} \lambda-4 i(-17+4 n) \omega\right)+r_{3-}{ }^{4} r_{3 c} \lambda\left(\left(-37+18 n-2 n^{2}\right) r_{3 c} \lambda\right.$

$+6 i(-39+10 n) \omega)+2 r_{3-}{ }^{3} r_{3 c}\left(-4\left(68-33 n+4 n^{2}\right) r_{3 c}{ }^{2} \lambda^{2}+3 i(-17+4 n) r_{3 c} \lambda \omega+36 \omega^{2}\right)+$

$\left.r_{3-}{ }^{2} r_{3 c}{ }^{2}\left(\left(-226+99 n-11 n^{2}\right) r_{3 c}{ }^{2} \lambda^{2}+24 i(-9+2 n) r_{3 c} \lambda \omega+72 \omega^{2}\right)\right)+r_{3+}{ }^{5}$

$\left(\left(215-95 n+11 n^{2}\right) r_{3-}{ }^{3} \lambda^{2}+r_{3-}{ }^{2} \lambda\left(\left(-945+460 n-56 n^{2}\right) r_{3 c} \lambda+6 i(-119+30 n) \omega\right)+\right.$

$3 r_{3-}\left(\left(3-5 n+n^{2}\right) r_{3 c}{ }^{2} \lambda^{2}-2 i(-133+34 n) r_{3 c} \lambda \omega-36 \omega^{2}\right)+r_{3 c}\left(7\left(23-14 n+2 n^{2}\right) r_{3 c}{ }^{2} \lambda^{2}\right.$

$\left.\left.-12 i(-7+2 n) r_{3 c} \lambda \omega+72 \omega^{2}\right)\right)+r_{3+}{ }^{3}\left(\left(-417+203 n-25 n^{2}\right) r_{3-}{ }^{5} \lambda^{2}-3 r_{3 c}{ }^{4} \lambda\left(\left(23-14 n+2 n^{2}\right)\right.\right.$

$\left.r_{3 c} \lambda-4 i(-7+2 n) \omega\right)+r_{3-}{ }^{4} \lambda\left(\left(865-434 n+54 n^{2}\right) r_{3 c} \lambda-6 i(-7+2 n) \omega\right)+2 r_{3-}{ }^{3}$ 


$$
\begin{aligned}
& \left(2\left(55-26 n+3 n^{2}\right) r_{3 c}{ }^{2} \lambda^{2}-9 i(-9+2 n) r_{3 c} \lambda \omega-36 \omega^{2}\right)+4 r_{3-}{ }^{2} r_{3 c}\left(\left(70-29 n+3 n^{2}\right) r_{3 c}{ }^{2} \lambda^{2}+\right. \\
& \left.\left.6 i(-4+n) r_{3 c} \lambda \omega+72 \omega^{2}\right)+r_{3-} r_{3 c}^{2}\left(\left(1+13 n-3 n^{2}\right) r_{3 c}{ }^{2} \lambda^{2}+144 i(-4+n) r_{3 c} \lambda \omega+216 \omega^{2}\right)\right)+ \\
& r_{3+}{ }^{4}\left(\left(-520+263 n-33 n^{2}\right) r_{3-}{ }^{4} \lambda^{2}+r_{3-}{ }^{3} \lambda\left(\left(-179+74 n-8 n^{2}\right) r_{3 c} \lambda+12 i(-33+8 n) \omega\right)+\right. \\
& r_{3-}{ }^{2}\left(\left(113-47 n+5 n^{2}\right) r_{3 c}{ }^{2} \lambda^{2}-6 i(-155+38 n) r_{3 c} \lambda \omega-180 \omega^{2}\right)+r_{3 c}{ }^{2}\left(\left(23-14 n+2 n^{2}\right)\right. \\
& \left.r_{3 c}^{2} \lambda^{2}+24 i(-7+2 n) r_{3 c} \lambda \omega+72 \omega^{2}\right)+r_{3-} r_{3 c}\left(\left(643-312 n+38 n^{2}\right) r_{3 c}{ }^{2} \lambda^{2}-6 i(-43+10 n)\right. \\
& \left.\left.\left.r_{3 c} \lambda \omega+288 \omega^{2}\right)\right)\right) \\
& \sigma_{n}=\frac{1}{\left(r_{3-}-r_{3 c}\right)^{5}} r_{3-}^{2}\left(r_{3+}-r_{3 c}\right)^{5}\left(-(-5+n)^{2}\left(r_{3+}-r_{3-}\right)\left(r_{3+}-r_{3 c}\right)^{2}\left(r_{3-}-r_{3 c}\right)\right. \\
& \left(2 r_{3+}+r_{3-}+r_{3 c}\right)^{2}\left(r_{3+}+2 r_{3-}+r_{3 c}\right) \lambda^{2}-6 i(-5+n)\left(r_{3+}-r_{3 c}\right)\left(4 r_{3+}{ }^{4}\left(r_{3-}-r_{3 c}\right)\right. \\
& +r_{3-}{ }^{2} r_{3 c}\left(r_{3-}+r_{3 c}\right)^{2}+r_{3+}{ }^{3}\left(6 r_{3-}{ }^{2}-4 r_{3-} r_{3 c}-6 r_{3 c}{ }^{2}\right)-2 r_{3+}{ }^{2} r_{3 c}\left(-r_{3-}{ }^{2}+2 r_{3-} r_{3 c}+r_{3 c}{ }^{2}\right)+ \\
& \left.r_{3+} r_{3-}{ }^{2}\left(-r_{3-}{ }^{2}+2 r_{3-} r_{3 c}+3 r_{3 c}{ }^{2}\right)\right) \lambda \omega+36 r_{3+}{ }^{2}\left(r_{3+}{ }^{2}\left(r_{3-}-r_{3 c}\right)-r_{3-} r_{3 c}\left(r_{3-}+r_{3 c}\right)\right. \\
& \left.\left.+r_{3+}\left(r_{3-}{ }^{2}-2 r_{3-} r_{3 c}-r_{3 c}^{2}\right)\right) \omega^{2}\right)
\end{aligned}
$$

[1] V.P. Frolov and I.D. Novikov, Black Hole Physics: Basic Concept and New Developments (Kluwer Academic, Dordrecht, 1998).

[2] D.T. Son and A.O. Starinets, Ann. Rev. Nucl. Part. Sci. 57, 95 (2007).

[3] B.P. Abbott et al. (LIGO Scientific Collaboration and Virgo Collaboration), Phys. Rev. Lett. 116, 061102 (2016); B.P. Abbott et al. (LIGO Scientific Collaboration and Virgo Collaboration), Phys. Rev. Lett. 116, 131103 (2016).

[4] R.A. Konoplya and R.D.B. Fontana, Phys. Lett. B 659, 375 (2008); R. Brito, V. Cardoso and P. Pani, Phys. Rev. D 89, 104045 (2014); C.Y. Zhang, S.J. Zhang, and B. Wang, Nucl. Phys. B 899, 37 (2015); M. Richartz and D. Giugno, Phys. Rev. D 90, 124011 (2014); R.A. Konoplya and A.V. Zhidenko, Phys. Lett. B 609, 377 (2005); S. Hod, Phys. Rev. D 91, 044047 (2015); R. Brito, V. Cardoso, P. Pani, arXiv: 1501.06570v2; E. Berti, V. Cardoso, and S. Yoshida, Phys. Rev. D 69, 124018 (2004); E. Betti and K.D. Kokkotas, Phys. Rev. D 67, 064020 (2003); H.T. Cho, A.S. Cornell, J. Doukas, T.R. Huang, and W. Naylor, Adv. Math. Phys. 2012, 281705 (2012); C. Wu and R. Xu, Eur. Phys. J. C. 75, 391 (2015); Z. Zhu, S.J. Zhang, C.E. Pellicer, B. Wang and E. Abdalla, Phys. Rev. D 90, 044042 (2014). 
[5] K.D. Kokkotas and B.G. Schmidt, Living Rev. Rel. 2, 2 (1999); H.P. Nollert, Class. Quantum Grav. 16, R159 (1999); E. Berti, V. Cardoso, and A.O. Starinets, Class. Quantum Grav. 26, 163001 (2009); R.A. Konoplya and A.V. Zhidenko, Rev. Mod. Phys. 83, 793 (2011); B. Wang, Braz. J. Phys. 35, 1029 (2005).

[6] S. Perlmutter et al., Astrophys. J. 517, 565 (1999).

[7] P. De Bernardis et al., Nature 404, 995 (2000); R. Lamon and R. Durrer, Phys. Rev. D 73, 023507 (2006).

[8] M. Tegmark et al., Phys. Rev. D 69, 103501 (2004).

[9] R.R. Caldwell, R. Dave, P.J. Steinhardt, Phys. Rev. Lett. 80, 1582 (1998); I. Zlatev, L. Wang, and P.J. Steinhardt, Phys. Rev. Lett. 82, 896 (1999); M. Doran and J. Jaeckel, Phys. Rev. D 66, 043519 (2002).

[10] B. Mcinnes, J. High Energy Phys. 0208, 029 (2002);

[11] J.H. He, B. Wang, and E. Abdalla, Phys. Lett. B 671, 139 (2009); J.H. He, B. Wang, and E. Abdalla, Phys. Rev. D 83, 063515 (2011).

[12] V.V. Kiselev, Class. Quantum Grav. 20, 1187 (2003).

[13] S. Chen and J. Jing, Class. Quantum Grav. 22, 4651 (2005);

[14] G. Guo, Eur. Phys. J. C. 73, 2573 (2013); Y. Zhang and Y.X. Gui, Class. Quantum Grav. 23, 6141 (2006); N. Varghese and V. C. Kuriakose, Gen. Relativ. Gravit. 45, 189 (2013).

[15] N. Varghese and V.C. Kuriakose, Gen. Relativ. Gravit. 41, 1249 (2009); M. Saleh, B. B. Thomas, and T. C. Kofane, Astrophys. Space Sci. 333, 449 (2011).

[16] E.M. Leaver, Proc. R. Soc. A 402, 285 (1995).

[17] R. A. Konoplya and A. Zhidenko, Phys. Rev. D 76, 084018 (2007).

[18] R.A. Konoplya, Phys. Rev. D 66, 084007 (2002). 
TABLE I: Fundamental quasinormal modes for Reissner-Nordström spacetime surrounded by quintessence for different values of $Q, l, c$ and $\epsilon$.

\begin{tabular}{|c|c|c|c|c|c|}
\hline$Q$ & $c$ & $l$ & $\omega(\epsilon=-1 / 3)$ & $\omega(\epsilon=-2 / 3)$ & $\omega(\epsilon=-1)$ \\
\hline \multirow[t]{3}{*}{0.01} & 0.001 & 0 & $0.110347-0.104792 \mathrm{i}$ & $0.109595-0.104484 \mathrm{i}$ & $0.108712-0.10470 \mathrm{i}$ \\
\hline & & 1 & $0.292648-0.0975628 \mathrm{i}$ & $0.291443-0.0971104 \mathrm{i}$ & $0.288213-0.096783 \mathrm{i}$ \\
\hline & & 2 & $0.483168-0.0966625 \mathrm{i}$ & $0.481362-0.0961919 \mathrm{i}$ & $0.47655-0.0956244 \mathrm{i}$ \\
\hline \multirow[t]{3}{*}{0.01} & 0.005 & 0 & $0.109905-0.104372 \mathrm{i}$ & $0.106146-0.102819 \mathrm{i}$ & $0.101025-0.104226 \mathrm{i}$ \\
\hline & & 1 & $0.291476-0.0971722 \mathrm{i}$ & $0.285418-0.0948987 \mathrm{i}$ & $0.268712-0.0928568 \mathrm{i}$ \\
\hline & & 2 & $0.481234-0.0962755 \mathrm{i}$ & $0.47214-0.0939135 \mathrm{i}$ & $0.447269-0.0907654 \mathrm{i}$ \\
\hline \multirow[t]{3}{*}{0.1} & 0.001 & 0 & $0.110538-0.104833 \mathrm{i}$ & $0.109786-0.104526 \mathrm{i}$ & $0.108907-0.104743 \mathrm{i}$ \\
\hline & & 1 & $0.293138-0.0976132 \mathrm{i}$ & $0.291933-0.0971613 \mathrm{i}$ & $0.288712-0.0968357 \mathrm{i}$ \\
\hline & & 2 & $0.483972-0.0967143 \mathrm{i}$ & $0.482165-0.0962442 \mathrm{i}$ & $0.477367-0.0956793 \mathrm{i}$ \\
\hline \multirow[t]{3}{*}{0.1} & 0.005 & 0 & $0.110095-0.104413 \mathrm{i}$ & $0.106336-0.102863 \mathrm{i}$ & $0.101225-0.104276 \mathrm{i}$ \\
\hline & & 1 & $0.291963-0.0972222 \mathrm{i}$ & $0.285906-0.0949515 \mathrm{i}$ & $0.269247-0.0929212 \mathrm{i}$ \\
\hline & & 2 & $0.48203-0.0963269 \mathrm{i}$ & $0.472939-0.0939677 \mathrm{i}$ & $0.448139-0.0908347 \mathrm{i}$ \\
\hline \multirow[t]{3}{*}{0.5} & 0.001 & 0 & $0.115642-0.105644$ & $0.114891-0.105349 \mathrm{i}$ & $0.114134-0.105616 \mathrm{i}$ \\
\hline & & 1 & $0.30624-0.0986983 \mathrm{i}$ & $0.305043-0.0982617 \mathrm{i}$ & $0.302049-0.097982 \mathrm{i}$ \\
\hline & & 2 & $0.505437-0.0978431 \mathrm{i}$ & $0.503643-0.0973889 \mathrm{i}$ & $0.499189-0.09689 \mathrm{i}$ \\
\hline \multirow[t]{3}{*}{0.5} & 0.005 & 0 & $0.115156-0.105219 \mathrm{i}$ & $0.111400-0.103728 \mathrm{i}$ & $0.106548-0.105305 \mathrm{i}$ \\
\hline & & 1 & $0.304954-0.0982993 \mathrm{i}$ & $0.298939-0.0961056 \mathrm{i}$ & $0.283495-0.0943666 \mathrm{i}$ \\
\hline & & 2 & $0.503315-0.0974474 \mathrm{i}$ & $0.494287-0.0951677 \mathrm{i}$ & $0.471391-0.0924144 \mathrm{i}$ \\
\hline \multirow[t]{3}{*}{0.9} & 0.001 & 0 & $0.132172-0.102119 \mathrm{i}$ & $0.131432-0.10187 \mathrm{i}$ & $0.130244-0.102581 \mathrm{i}$ \\
\hline & & 1 & $0.352134-0.0971095 \mathrm{i}$ & $0.350982-0.0967346 \mathrm{i}$ & $0.348699-0.096565 \mathrm{i}$ \\
\hline & & 2 & $0.581213-0.0965454 \mathrm{i}$ & $0.579482-0.0961557 \mathrm{i}$ & $0.56217-0.0958204 \mathrm{i}$ \\
\hline \multirow[t]{3}{*}{0.9} & 0.005 & 0 & $0.131536-0.101798 \mathrm{i}$ & $0.127835-0.100546 \mathrm{i}$ & $0.123428-0.102363 \mathrm{i}$ \\
\hline & & 1 & $0.350353-0.0967819 \mathrm{i}$ & $0.344562-0.0948978 \mathrm{i}$ & $0.332872-0.093862 \mathrm{i}$ \\
\hline & & 2 & $0.578263-0.0962164 \mathrm{i}$ & $0.569551-0.0942599 \mathrm{i}$ & $0.55224-0.0925138 \mathrm{i}$ \\
\hline
\end{tabular}



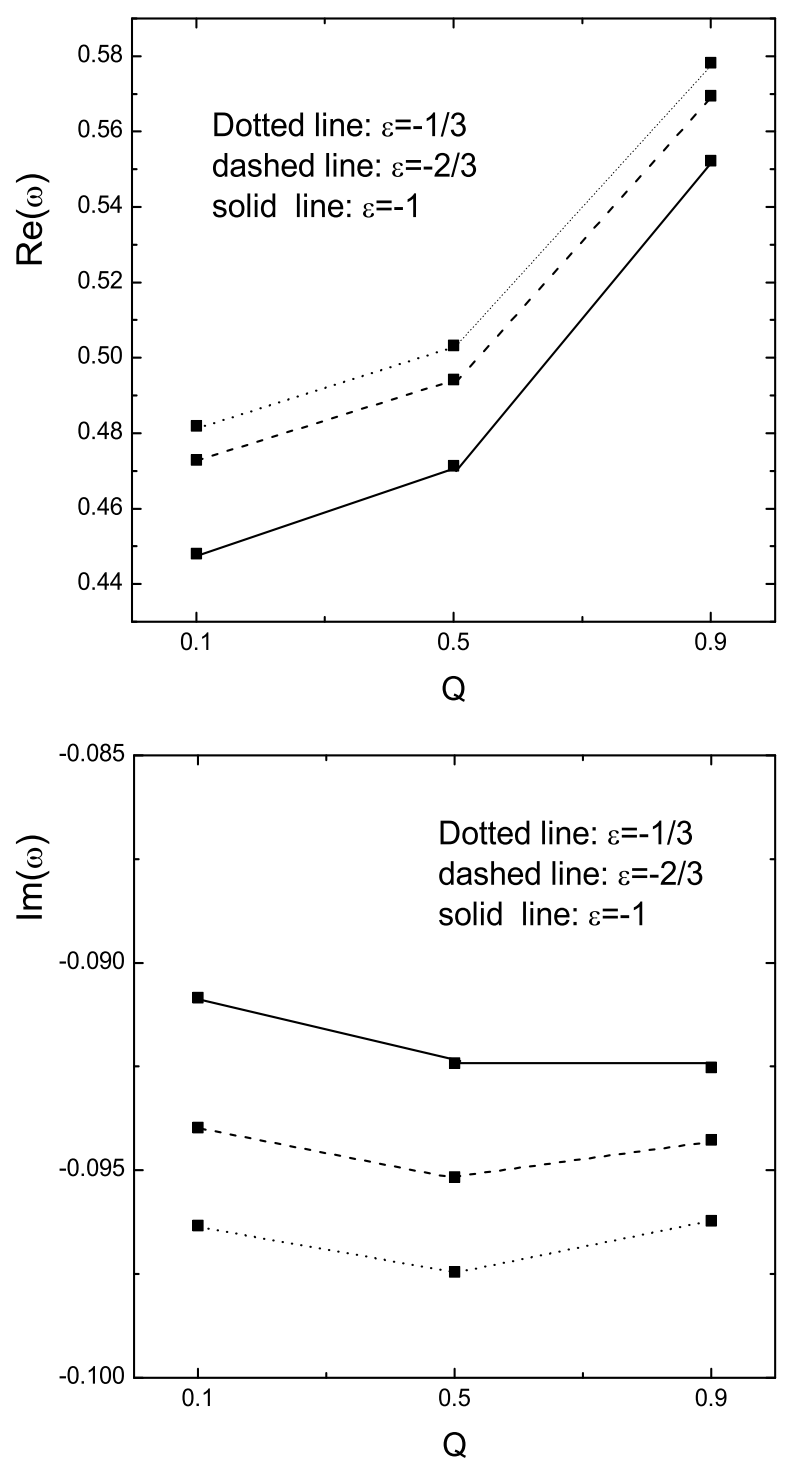

FIG. 1: $\operatorname{Re}(\omega)$ and $\operatorname{Im}(\omega)$ for scalar field in Reissner-Nordström spacetime surrounded by quintessence as a function of $Q$ for $l=2$ and for different values of $\epsilon$ with $c=0.005$. 

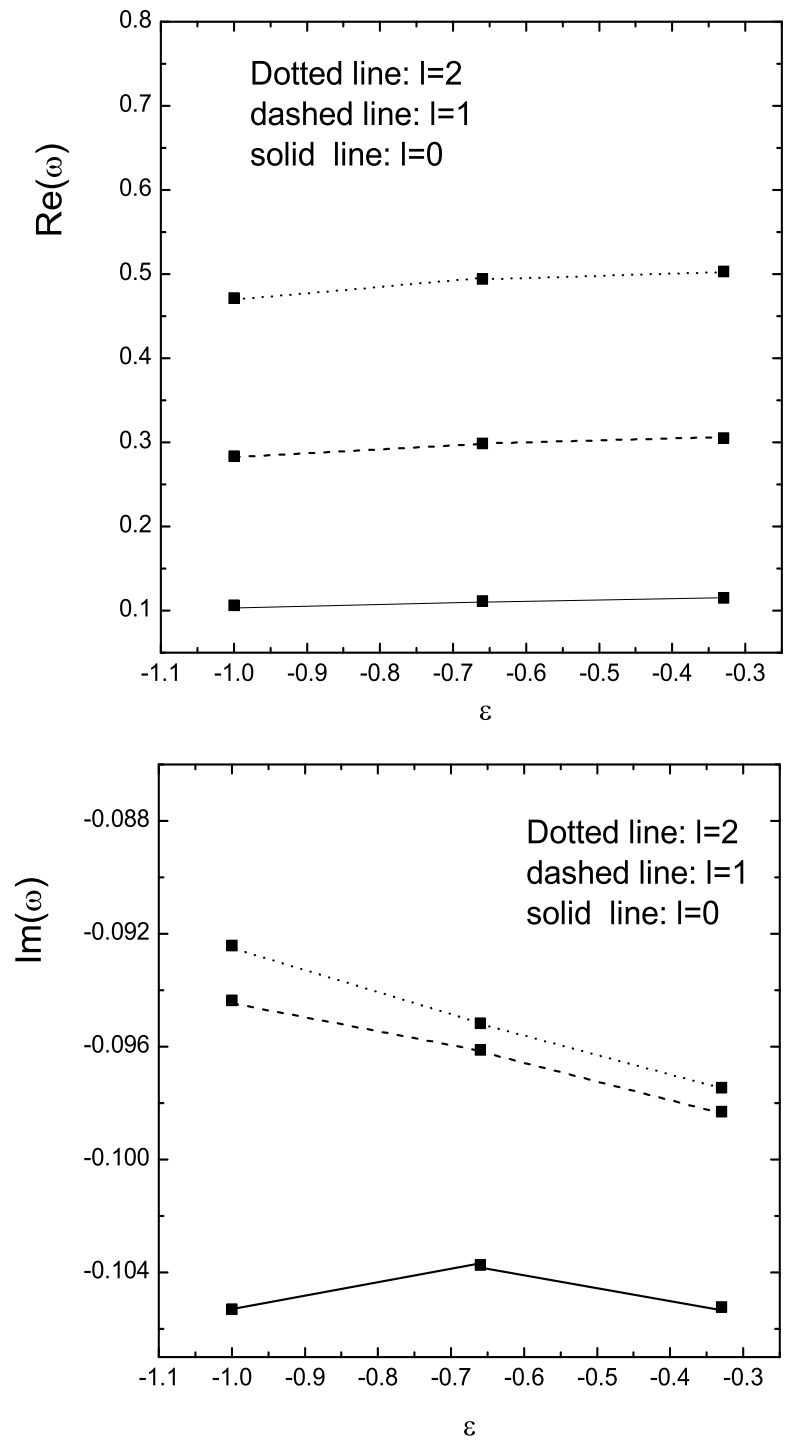

FIG. 2: $\operatorname{Re}(\omega)$ and $\operatorname{Im}(\omega)$ for scalar field in Reissner-Nordström spacetime surrounded by quintessence as a function of $\epsilon$ for $Q=0.5$ and $c=0.005$ and for different values of $l$. 